\title{
Abnormality in the cavernous sinus in three patients with Tolosa-Hunt syndrome: MRI and CT findings
}

\author{
Yoshinobu Goto, Shinichi Hosokawa, Ikuo Goto, Ryousuke Hirakata, Kanehiro Hasuo
}

\begin{abstract}
Three patients with Tolosa-Hunt syndrome (THS) were examined using computed tomography (CT), orbital venography and magnetic resonance imaging (MRI). CT of the brain showed no definite abnormality in two patients, right optic nerve enlargement and an abnormal area around the orbital apex in one patient. Orbital venography showed an occlusion of the superior ophthalmic vein in one of three patients. MRI showed an abnormal soft tissue area in the cavernous sinus, with intermediate to high signal intensity on T1- or intermediate weighted images. There was clinical improvement following corticosteroid therapy and the abnormal soft tissue on MRI decreased in volume and in signal intensity, although this was equivocal in one patient. Thus MRI proved to be useful in demonstrating lesions in the cavernous sinus in patients with THS.
\end{abstract}

The Tolosa-Hunt syndrome (THS) is a painful ophthalmoplegia due to non-specific granulomatous inflammation in the cavernous sinus or superior orbital fissure. ${ }^{1-3}$ The diagnosis is based on findings of painful ophthalmoplegia, excellent response to corticosteroid and exclusion of other causes, including aneurysm, diabetes mellitus, paranasal mucocoele, parasellar neoplasms, carotid cavernous fistula. Direct visualisation of lesions in the cavernous sinus in THS is difficult.

We report our findings in three patients with

Faculty of Medicine, Kyushu University, Fukuoka, Japan Department of Neurology

Y Goto*

$S$ Hosokawa

I Goto

Department of

Radiology

R Hirakata

K Hasuo

Department of Neurosurgery ${ }^{\star}$

Correspondence to: Dr Shinichi Hosokawa, Department of Neurology, Neurological Institute, Faculty of Medicine, Kyushu University 60, 3-1-1 Maidashi, Higashi-ku, Fukuoka 812, Japan.

Received 31 January 1989 and in revised form

29 May 1989.
Accepted 17 September 1989 THS. The abnormality in the cavernous sinus alised by magn (MRI). Other neuroradiological findings including computed tomography (CT) and orbital venography in these patients were also compared with those of MRI.

\section{Subjects and methods}

Three patients with THS were neuroradiologically studied, including plain skull radiography, CT, orbital venography and MRI. CT scans were performed with a highresolution mode of a $2 \mathrm{~mm}$ thickness as well as routine CT, with and without contrast. All MRI studies were performed on a high-field strength ( 1.5 tesla), super-conducting General Electric SIGNA MR imaging system. The T2weighted, spin echo (SE) images were obtained using a repetition time (TR) of $2000 \mathrm{~ms}$ and an echo time (TE) of $80 \mathrm{~ms}$ (SE 2000/80) in the axial and coronal planes. Intermediate weighted (SE 2000/20) images were obtained simultaneously. The T1-weighted, partial saturation (PS) images were also obtained for most patients (PS 600/25). Using a multislice technique, 19 images of SE 2000/80, 19 images of SE $2000 / 20$ and 9 images of PS $600 / 25$ were obtained with a $256 \times 256$ matrix and $5 \mathrm{~mm}$ slice thickness, and a $24 \mathrm{~cm}$ field of view for the coronal images and a $20 \mathrm{~cm}$ field of view for the axial images.

\section{Case report}

Case 1 A 63 year old man gradually developed numbness under the right eye six months before admission. He also had right proptosis and orbital pain five months later. There was a history of left trigeminal neuropathy 10 years before which was relieved by corticosteroid therapy. Examination showed anisocoria, slightly delayed light reflex, complete paralysis of ocular movement in all directions, absent corneal reflex and hypoaesthesia in the V1 and V2 distributions, all to the right side. Relevant laboratory data including haematology, erythrocyte sedimentation rate, serology and blood chemistry were normal and the plain skull radiograph was also normal. The brain CT suggested an enlarged cavernous sinus on the right. The orbital venography also was normal. The axial and coronal T1- and intermediate weighted images on MRI showed an abnormal soft tissue area with an intermediate signal intensity within the right cavernous sinus. This abnormal area was localised in the lateral to the right internal carotid artery (fig $1 \mathrm{~A}$ and $\mathrm{C}$ ).

A therapeutic trial of prednisolone was star- 
Figure 1 MRI of case 1. The axial and coronal images $(A, B$ :

intermediate, $C, D: T 1-)$ show abnormal soft tissue area in the right cavernous sinus before therapy $(A$, $C)$. The abnormal area decreased in volume and in signal intensity after therapy $(B, D)$.
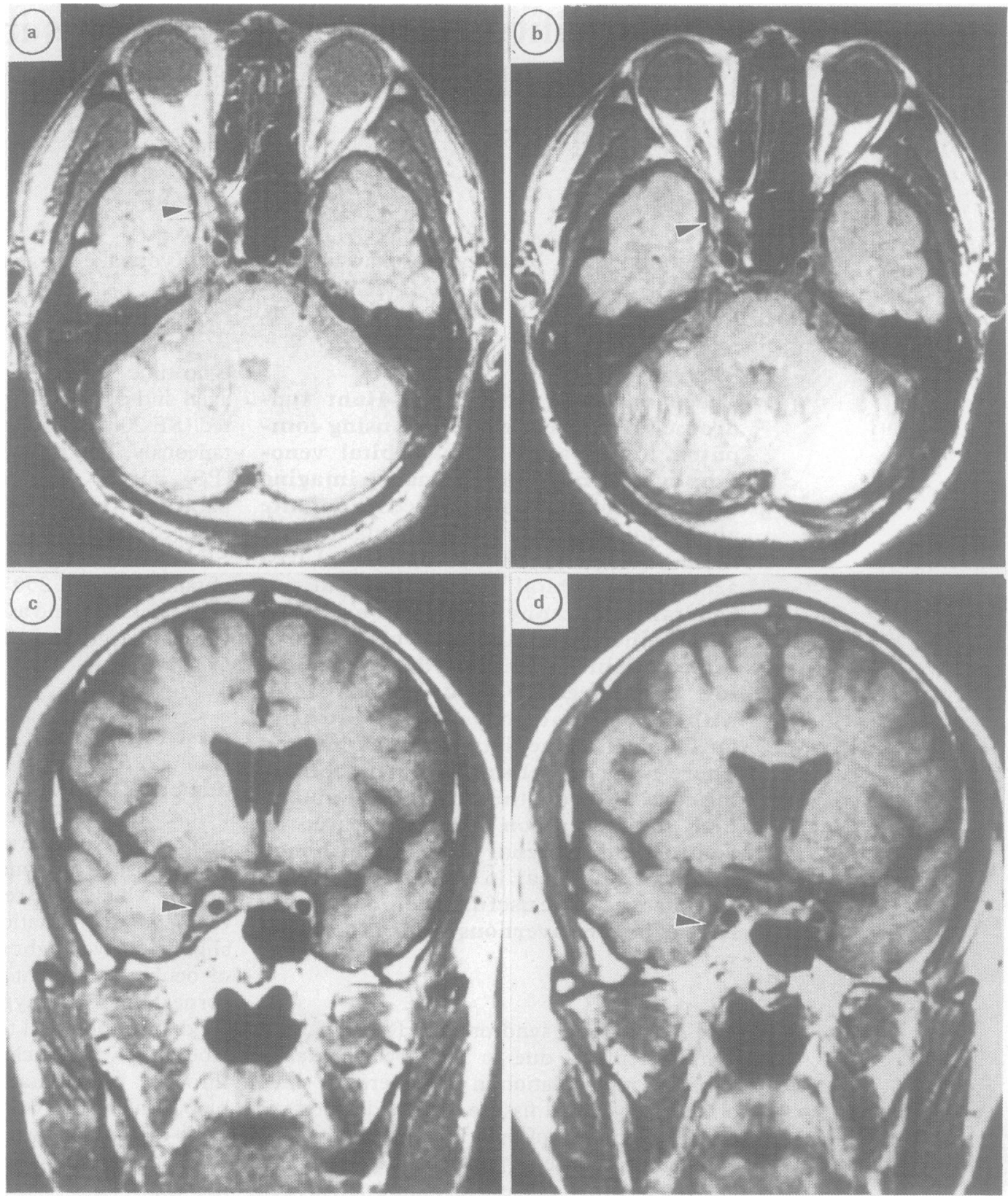

cyte sedimentation rate. The CT showed an enlarged optic nerve sheath and an abnormal soft tissue area around the orbital apex, on the right, but with no abnormality within the cavernous sinus. Orbital venography showed occlusion of the third segment of the right superior ophthalmic vein. The point of obstruction was bypassed through small collateral veins. On MRI, the T1-weighted images showed no definite abnormality, but intermediate weighted images revealed an abnormal soft tissue area with an intermediate to high signal intensity around the right orbital apex and within the cavernous sinus.

All signs and symptoms resolved except for a mild hypoaesthesia in V1 distribution after two weeks on corticosteroids. The follow up CT was normal and the MRI suggested a decrease in the abnormal areas.

Case 3 A healthy 49 year old man had acute onset of right orbital pain and had a tingling sensation on the right half of the face one month later. Subsequently, diplopia occurred with right lateral gaze. Physical examination showed a complete sixth nerve palsy, hypoaesthesia in $\mathrm{V} 1$ and V2 distributions and a delayed corneal reflex, all on the right. All the laboratory data were normal except for a slight increase in the erythrocyte sedimentation rate. The plain skull radiograph and CT were normal throughout graphy was also normal. The axial and coronal T1- and intermediate weighted images on MRI showed an abnormal soft tissue area with intermediate signal intensity within the right cavernous sinus (fig 2A, C). The abnormal area was mainly at the lower area of the internal carotid artery.

Corticosteroid therapy was effective in relieving signs and symptoms. The abnormal area on MRI decreased in volume and the signal intensity dropped after corticosteroid therapy (fig 2B, D), in both the axial and coronal images.

A summary of the clinical and neuroradiological findings in the three patients is given in the table. the course of the disease. The orbital veno- 
Figure 2 MRI of case 3. The axial and coronal images ( $A, B: T 1-, C, D$ : intermediate) show abnormal soft tissue area in the right cavernous sinus before therapy $(A, C)$. It decreased in volume after therapy $(B, D)$.

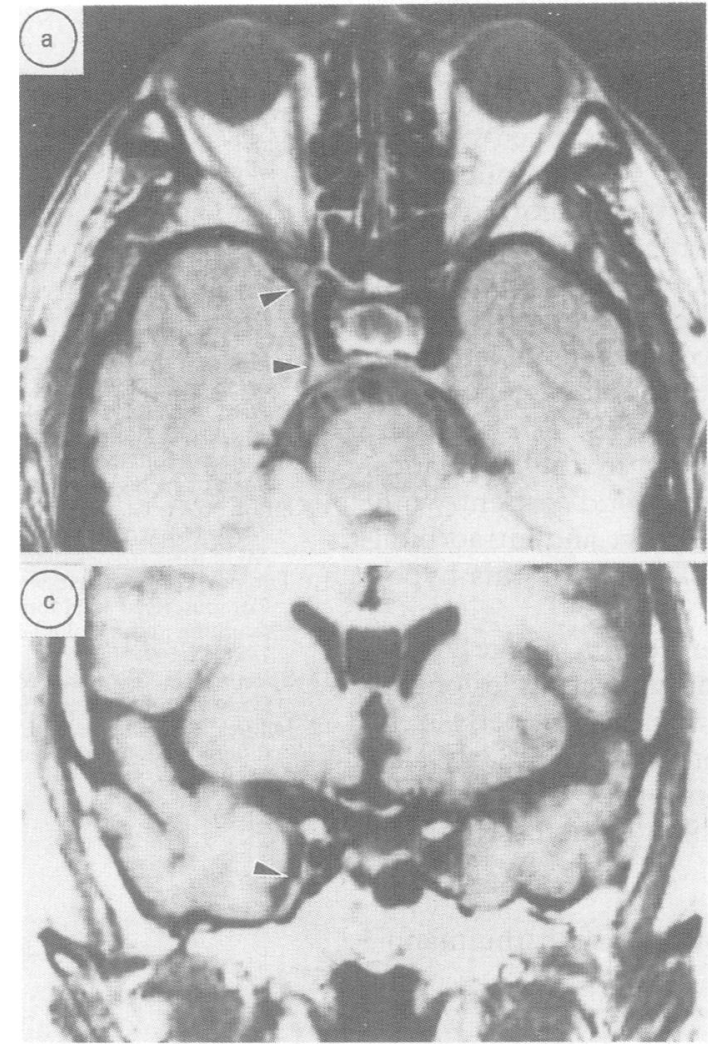

Table Summary of clinical and neuroradiological findings in three patients with Tolosa-Hunt syndrome

\begin{tabular}{llll}
\hline & Case 1 & Case 2 & Case 3 \\
\hline $\begin{array}{llll}\text { Age (years)/Sex } \\
\text { Past History }\end{array}$ & $\begin{array}{l}\text { 63/Male } \\
\text { Trigeminal neuropathy }\end{array}$ & $\begin{array}{l}55 / \text { Female } \\
\text { nc }\end{array}$ & $\begin{array}{l}\text { 49/Male } \\
\text { nc }\end{array}$ \\
Onset & (L) & Acute & Acute \\
Side & Subacute & Right & Right \\
Symptoms & $\begin{array}{l}\text { Right } \\
\text { Orbital pain }\end{array}$ & $\begin{array}{l}\text { Orbital pain } \\
\text { Diplopia }\end{array}$ & $\begin{array}{l}\text { Orbital pain } \\
\text { Diplopia }\end{array}$ \\
& Facial numbness & Diplopia & Tingling sensation of \\
Cranial nerve palsies & III, IV, VI, V1, V2, & III, VI, V1 & VI, V1, V2 \\
& $\begin{array}{l}\text { Anisocoria, corneal } \\
\text { reflex: absent }\end{array}$ & Proptosis & $\begin{array}{l}\text { Corneal reflex: } \\
\text { delayed }\end{array}$ \\
Efficacy of steroid & $(+)$ & $(+)$ & $(+)$ \\
CT & Equivocal & Abnormal & n \\
Sup ophthal vein occl & $(-)$ & $(+)$ & $(-)$ \\
MRI abnormality & $(+)$ & $(+)$ & $(+)$ \\
\hline
\end{tabular}

nc: non-contributory; n: normal; sup ophthal vein occl: superior ophthalmic vein occlusion.

\section{Discussion}

Our three patients had unilateral painful ophthalmoplegia, which was steroid-responsive. The presence of other disease was excluded and these patients were diagnosed as having the Tolosa-Hunt syndrome. This syndrome is caused by a non-specific inflammatory process in the cavernous sinus or superior orbital fissure. ${ }^{1-3}$ Orbital venography is helpful to prove occlusion of the superior ophthalmic vein, but it is difficult to visualise the lesion in the cavernous sinus. ${ }^{5}$ CT scans were normal in the majority of patients with THS.$^{67}$ It has been reported recently that highresolution CT can show abnormal soft tissue area in both the cavernous sinus and orbital apex in patients with THS ${ }^{8}$ Nevertheless, the limitation of contrast resolution due to lack of sensitivity to soft tissue change with superimposed beam hardening and bony streak artefacts makes it difficult to accurately visualise lesions in the cavernous sinus. MRI can reveal

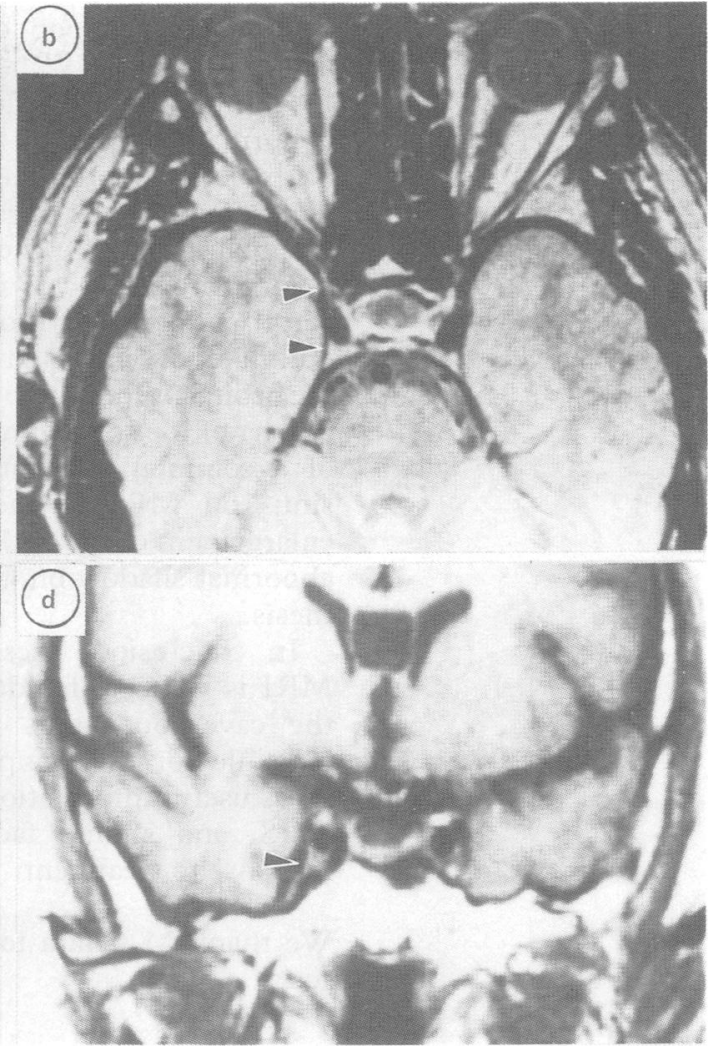

localised minute lesions in the brain and is suited for assessing lesions surrounded by bone such as the cavernous sinus and orbit. ${ }^{9}$ There have been few reports on MRI findings in patients with THS. ${ }^{810}$ Since this study reports MRI findings in only a small number of patients with THS seen by us, the incidence rate of abnormality on MRI in patients with THS cannot be determined. However, the demonstration of abnormal soft tissue area in the cavernous sinus of all three patients with THS, without any definite abnormality seen on CT shows that MRI will be invaluable in demonstrating lesions that are directly responsible for the symptoms of THS. The abnormal area in the cavernous sinus on MRI in our patients, seen as an intermediate signal intensity on T1- and intermediate weighted images, is consistent with the pathological process of THS, that is, granulomatous inflammation. The abnormal area on MRI decreased in volume and in signal intensity after corticosteroid therapy improved clinical symptoms, although this was doubtful in one patient. This improvement in MRI findings gives additional information in monitoring the response to treatment in patients with THS.

The location of an abnormality reflects that of the involved cranial nerves in the cavernous sinus. Anatomically, it is well known that the oculomotor (III) and trochlear (IV) nerves are upper and lateral to the internal carotid artery in the cavernous sinus, and the abducens nerve (VI) and the ophthalmic (V1) and maxillary (V2) divisions of trigeminal nerve are lower to that. ${ }^{11}$ In patient 1 , with mainly cranial nerves III and IV involved, the abnormality on MRI was located lateral to the internal carotid artery. In patient 3 , with involvement of cranial nerves VI, V1 and V2, the abnormal shadow was 
inferior to the loop of the internal carotid artery.

Painful ophthalmoplegia with an intraorbital abnormal shadow on CT has been termed "orbital pseudotumour". ${ }^{12}$ Our unpublished data show that the intraorbital abnormality on CT may be due to the relatively long-lasting inflammatory process itself and/or oedema in soft tissues, caused by occlusion of the superior ophthalmic vein or cavernous sinus and that the orbital pseudotumour should be considered as part of the spectrum of THS. The presence of a common abnormality in the cavernous sinus on MRI in the second patient with enlargement of the optic nerve and intraorbital abnormal shadow on CT supports this hypothesis.

In conclusion, these results suggest that MRI is valuable for demonstrating lesions in the cavernous sinus that are directly responsible for the symptoms of THS. It also gives useful information for the diagnosing of THS, and should facilitate monitoring the response to treatment.

We thank M Ohara for help with the manuscript.
1 Hunt WE, Meagher JN, LeFever HE, Zeman W. Painful ophthalmoplegia. Its relation to indolent inflammation of the cavernous sinus. Neurology 1961;11:56-62.

2 Hunt WE. Tolosa-Hunt syndrome: One cause of painful ophthalmoplegia. J Neurosurg 1976;44:544-9.

3 Tolosa E. Periarteritic lesions of the carotid siphon with the clinical features of a carotid infraclinoid aneurysm. $J$ Neurol Neurosurg Psychiatry 1954;17:300-2.

4 Kline LB. The Tolosa-Hunt syndrome. Surv Ophthalmol 1982;27:79-95.

5 Muhletaler CA, Gerlock AJ Jr. Orbital venography in painful ophthalmoplegia (Tolosa-Hunt syndrome). Am J Radiol 1979;133:31-4

6 Aron-Rosa D, Doyon D, Salamon G, Michotey P. TolosaHunt syndrome. Ann Ophthalmol 1978;10:1161-8.

7 Van Dalen JTW, Bleeker GM. The Tolosa-Hunt syndrome. Doc Ophthalmol 1977;44:167-72.

8 Kwan ESK, Wolpert SM, Hedges III TR, Laucella ML. Tolosa-Hunt syndrome revisited: not necessarily a diagnosis of exclusion. Am J Neuroradiol 1987;8:1067-72.

9 Smith FW, Cherryman GR, Singh AK, Forrester JV. Nuclear magnetic resonance tomography of the orbit at 3.4 MHz. Br J Radiol 1985;58:947-57.

10 Thomas DJB, Charlesworth MC, Afshar F, Galton DJ. Computerised axial tomography and magnetic resonance scanning in the Tolosa-Hunt syndrome. Br J Ophthalmol 1988;72:299-302.

11 Spector RH, Fiandaca MS. The "sinister" Tolosa-Hunt syndrome. Neurology 1986;36:198-203.

12 Jellinek EH. The orbital pseudotumour syndrome and its differentiation from endocrine exophthalmos. Brain 1969;92:35-58. 